\title{
Can I drive after those drops, doctor?
}

Abstract

Purpose To document the experience of patients driving after drops to dilate the pupils. There are no documented guidelines on driving after pupillary dilatation. This is a study of patients who drove a car after attending the eye casualty for an ocular examination during which their pupils were dilated.

Methods Thirty patients were identified who felt confident to drive after their pupils were dilated. Distant visual acuities, colour vision and confrontation visual fields were assessed before and after pupillary dilatation with $2.5 \%$ phenylephrine and $1 \%$ tropicamide. These patients met the legal requirements for driving after pupillary dilatation. The patients completed a questionnaire that recorded their subjective experiences of driving while their pupils were dilated.

Results The mean age of the patients was $51.9 \pm 19.7$ years (range 20-73 years). Twentyseven of the 30 patients undertook the journey on familiar roads, and 14 of 30 wore spectacles. The mean spherical equivalent was $+\mathbf{2 . 0 0}$ dioptre sphere for distance and a mean additional spherical equivalent of 1.75 dioptre sphere for near. Twenty patients experienced glare, which was severe enough to cause difficulty driving in three cases. Two of these patients drove in sunny weather conditions and one in light (not sunny) weather. Six patients had difficulty with road signs, two had difficulty judging distances and one reported difficulty with traffic lights.

Conclusions Dilating the pupils did not reduce distance visual acuity. However, it would seem appropriate to warn patients of the problems associated with glare and, if driving is their only option for transport home, recommend that they allow sufficient time to adapt to a dilated pupillary state, drive only on familiar roads and avoid driving in sunny weather.

Key words Driving, Dilating drops

Pupillary dilatation is commonly performed for fundal examination during emergency casualty visits to eye departments. A dilemma that frequently confronts the patient is whether they will be able to drive after their pupils have been dilated and whether they meet the legal requirements for driving when pupils are dilated. There are no documented guidelines on this issue. It is surmised by some that the distance vision is not affected and hence patients can drive, while others feel that the acute visual disturbance due to the dilated pupils with its attendant problems of spherical and chromatic aberrations, glare and a reduced visual acuity make it unsafe to drive.

We studied the subjective effects of pupillary dilatation in people who presented to the eye casualty department, and for whom the only means of transport after their eyes had been examined was the vehicle in which they had driven to the hospital.

\section{Materials and methods}

Patients who had driven to hospital for a consultation as an emergency visit and who required their pupils to be dilated as part of the ocular examination were recruited for this study. The inclusion criteria are listed in Table 1.

The majority of patients presented with a history of floaters (Table 2). Before dilating drops were instilled and before undertaking the journey the patients were warned that there would be an unquantified amount of glare and that reading the figures on the dashboard might not be possible. Thirty patients who had had both their pupils dilated with $2.5 \%$

phenylephrine and 1\% tropicamide for fundoscopy agreed to fill in a questionnaire (Table 3) about their experiences when driving with their pupils dilated. A reply-paid envelope was provided for return of the completed questionnaire. The patients who met the legal criteria for driving after dilating drops had been instilled were advised to drive only if they felt comfortable in doing so.

The patients' Snellen visual acuities (monocular and binocular), colour vision (tested with Ishihara colour plates) and confrontation fields (recording the outer limit at which a white target first appeared in the temporal, nasal superior and inferior field) were measured both before and after pupillary dilatation. The sizes of the dilated pupils just before the patient undertook the journey home and the colour of the irides were recorded.

\author{
P. Watts \\ D. O'Duffy \\ S. McCleod \\ S. L. Watson \\ Prince Charles Eye Unit \\ King Edward VII Hospital \\ Windsor SL4 3DP, UK \\ D. O'Duffy \\ C. Riddell \\ Royal Berkshire Hospital \\ Reading, UK \\ Mr Patrick Watts \\ 58 Crystal Wood Road \\ Heath \\ Cardiff CF4 4HW, UK
}




\section{Table 1. Inclusion criteria}

The patient's only means of transport was the vehicle (motor car) they had driven to hospital in

The patient met the legal requirements for driving after the drops took effect (Snellen acuity of better than 6/12)

The patient felt confident to drive after his or her pupils were dilated

\section{Results}

The patients' mean age was $51.9 \pm 19.7$ years (range 20-73 years); there were 18 men and 12 women. The mean driving experience was $31.3 \pm 11.8$ years. Twentyseven patients undertook the journey on familiar roads and 14 wore spectacles. The mean spherical equivalent was +2.00 dioptres with an additional reading add of +1.75 dioptres. The mean time from the drops being instilled to undertaking the journey home was $64.1 \pm 46.9 \mathrm{~min}$ (range 30-210 $\mathrm{min}$ ). Twenty patients experienced glare; however, only 3 of these had problems driving. There were 19 patients who undertook their journey in light conditions (not sunny), while 3 drove in bright sunny conditions and 8 drove in the dark.

Six patients had difficulty reading road signs, 2 had difficulty judging distances and 1 patient mentioned difficulty with traffic lights; this last patient also had problems with glare and judging distances but did not report any problems in his ability to complete his journey (Table 4).

Of the 3 patients who reported difficulty driving, one was a 48-year-old male emmetropic for distance who drove in bright weather conditions, another was a 54-year-old woman with a refractive error of +1.50 dioptre sphere (spherical equivalent) for distance and +1.50 dioptre sphere (spherical equivalent) for near who drove in light weather conditions, and the third was a 45-year-old man with no refractive error for distance who drove in bright weather conditions.

When the visual acuity was considered, one patient dropped 2 lines of binocular Snellen acuity. He was a 34-year-old man screened for diabetic retinopathy; he had a normal fundus with no evidence of diabetic retinopathy. Ten patients dropped 1 line of binocular Snellen visual acuity and 19 patients retained the same binocular Snellen visual acuity after their pupils were dilated. The patients' details are listed in Table 5 .

Table 2. Presenting complaints/diagnosis



Table 3. The patients' questionnaire

1. For how many years have you been driving?

2. Was the drive home on familiar roads?

3. What was the time interval between the drops being instilled and driving away from the clinic?

4. What were the weather conditions at the time (sunny, light, dark, rain, etc.)?

5. Was there any subjective glare?

6. Was there difficulty with road signs?

7. Was there difficulty judging distances?

8 . Were there any problems with traffic lights?

9. Did you wear spectacles/sunglasses while driving?

10. Any other comments?

\section{Discussion}

A person driving a motor vehicle is required by law to be able to read in good daylight a registration plate fixed to a motor vehicle containing letters and figures $79.4 \mathrm{~mm}$ high ( $3 \frac{1}{8}$ inches) at a distance of $20.5 \mathrm{~m} .{ }^{1}$ This equates to a Snellen visual acuity of $6 / 10 .^{2}$ This level of visual acuity is used by clinicians to advise patients about their fitness to drive if they have a standard of minimum field of vision. There are no guidelines on driving after dilating drops have been instilled in the eye and clinicians' advice may vary.

Though dilatation of the pupils did not significantly affect the level of binocular visual acuity, this test was done indoors and hence does not take into account the amount of glare that may be caused in bright daylight conditions. The use of a glare tester may have added useful information as to the amount of glare experienced with dilated pupils. None of the patients who drove in the dark experienced difficulty driving, though 2 of 8 reported glare.

\section{Conclusions}

Advising people whether or not to drive after dilating drops is a difficult subject. Though the patients selected in this study may not be representative of all patients attending the eye clinic casualty department we feel that a blanket rule of not driving after pupillary dilatation

Table 4. Summary of results

Age: $51.9 \pm 19.7$ years (range $20-73$ years)
Sex: 18 men, 12 women
Iris colour: 19 light, 11 dark
Pupil diameter: $7.3 \pm 0.8 \mathrm{~mm}$ (range $5-9 \mathrm{~mm}$ )
Prescription spectacles: 14 patients (mean spherical equivalent
+2.00, range $1-2.75$ )
Years of driving experience: $31.3 \pm 11.8$ years (range $3-55$ years)
Driving on familiar roads: 27 patients
Time of undertaking journey after drops: $64.1 \pm 46.9$ min (range
30-210 min)
Weather conditions: 22 patients in the light ( $3 / 22$ sunny), 8 in
the dark
Binocular visual acuity after drops: $6 / 9$ or better all cases
Drop in binocular visual acuity after drops: 1 patient, 2 Snellen
lines; 10 patients, 1 Snellen line
Glare: 20 patients
Difficulty with road signs: 6 patients
Difficulty judging distances: 2 patients
Difficulty with traffic lights: 1 patient




\begin{tabular}{|c|c|c|c|c|c|c|c|c|c|c|c|c|c|c|c|c|c|c|c|c|c|}
\hline \multirow[b]{2}{*}{$\begin{array}{l}\text { Patient } \\
\text { no. }\end{array}$} & \multirow{2}{*}{$\begin{array}{c}\text { Age } \\
\text { (years) } \\
\text { and sex }\end{array}$} & \multirow{2}{*}{$\begin{array}{c}\text { Years } \\
\text { driving }\end{array}$} & \multirow[b]{2}{*}{$\begin{array}{l}\text { Familiar } \\
\text { roads }\end{array}$} & \multirow{2}{*}{$\begin{array}{c}\text { Spectacles } \\
\text { used }\end{array}$} & \multirow{2}{*}{$\begin{array}{c}\text { Time of } \\
\text { driving } \\
\text { after drops } \\
\text { (min) }\end{array}$} & \multirow{2}{*}{ Conditions } & \multirow[b]{2}{*}{ Glare } & \multirow[b]{2}{*}{$\begin{array}{l}\text { Road } \\
\text { signs }\end{array}$} & \multirow[b]{2}{*}{$\begin{array}{l}\text { Judging } \\
\text { distance }\end{array}$} & \multirow[b]{2}{*}{$\begin{array}{l}\text { Traffic } \\
\text { lights }\end{array}$} & \multirow{2}{*}{$\begin{array}{c}\text { Any } \\
\text { problems } \\
\text { driving }\end{array}$} & \multicolumn{2}{|c|}{ VA before } & \multirow{2}{*}{$\begin{array}{c}\text { BVA } \\
\text { before }\end{array}$} & \multicolumn{2}{|c|}{ VA after } & \multirow{2}{*}{$\begin{array}{l}\text { BVA } \\
\text { after }\end{array}$} & \multirow{2}{*}{$\frac{\mathrm{CV}}{\text { before after }}$} & \multirow{2}{*}{$\begin{array}{l}\text { Pupil } \\
\text { diameter } \\
(\mathrm{mm})\end{array}$} & \multirow{2}{*}{$\begin{array}{c}\text { Iris } \\
\text { colour }\end{array}$} & \multirow[b]{2}{*}{ Diagnosis } \\
\hline & & & & & & & & & & & & $\mathrm{R}$ & $\overline{\mathrm{L}} \mathrm{b}$ & & $\mathrm{R}$ & $\mathrm{L}$ & & & & & \\
\hline 1 & $48 \mathrm{M}$ & 30 & Yes & Dark & 60 & Bright sun & + & - & - & - & Yes & $6 / 6$ & $6 / 6$ & $6 / 5$ & $6 / 6$ & $6 / 6$ & $6 / 6$ & $14 / 1714 / 17$ & $8 / 9$ & Blue & PVD \\
\hline 2 & $59 \mathrm{~F}$ & 30 & Yes & None & 60 & light-pm & + & - & - & - & No & $6 / 5$ & $6 / 5$ & $6 / 5$ & $6 / 6$ & $6 / 9$ & $6 / 6$ & $14 / 1714 / 17$ & 7 & Blue & PVD \\
\hline 3 & $51 \mathrm{M}$ & 34 & No & None & 30 & light-pm & + & - & - & - & $\begin{array}{l}\text { No- } \\
\text { caution }\end{array}$ & $6 / 6$ & $6 / 6$ & $6 / 6$ & $6 / 6$ & $6 / 6$ & $6 / 6$ & $15 / 17$ 15/17 & 7 & Blue & PVD \\
\hline 4 & $72 \mathrm{M}$ & 43 & Yes & None & 30 & light-pm & + & + & - & - & No & $6 / 9$ & $6 / 12$ & $6 / 9$ & $6 / 9$ & $6 / 12$ & $6 / 9$ & $15 / 1715 / 17$ & 5.7 & Blue & PVD \\
\hline 5 & $54 \mathrm{~F}$ & 34 & Yes & $\begin{array}{l}\text { p1.50ds } \\
\text { p1.5ad }\end{array}$ & 180 & light-am & + & - & - & - & Yes & $6 / 5$ & $6 / 9$ & $6 / 5$ & $6 / 6$ & $6 / 12$ & $6 / 6$ & $15 / 17$ 15/17 & $7 / 8$ & Brown & Glaucoma \\
\hline 6 & $48 \mathrm{M}$ & 30 & Yes & $\begin{array}{l}\text { p1.00ds } \\
\text { p1.00ad }\end{array}$ & 40 & light-pm & + & - & - & - & No & $6 / 6$ & $6 / 6$ & $6 / 6$ & $6 / 6$ & $6 / 9$ & $6 / 6$ & $14 / 1714 / 17$ & 8 & Grey & I uveitis \\
\hline 7 & $50 \mathrm{M}$ & 32 & Yes & $\begin{array}{l}\text { p1.75ds } \\
\text { p1.50ad }\end{array}$ & 90 & light-pm & + & + & - & - & No & $6 / 6$ & $6 / 9$ & $6 / 6$ & $6 / 6$ & $6 / 9$ & $6 / 6$ & $15 / 1715 / 17$ & 8 & Blue & PVD \\
\hline 8 & $34 \mathrm{M}$ & 17 & Yes & none & 60 & dark-pm & + & + & - & - & No & $6 / 5$ & $6 / 5$ & $6 / 5$ & $6 / 18$ & $6 / 18$ & $6 / 12$ & $15 / 1715 / 17$ & 8 & Blue & D ref \\
\hline 9 & $66 \mathrm{M}$ & 35 & Yes & $\begin{array}{l}\text { p2.00/ } \\
\text { p2.00ad }\end{array}$ & 30 & light-am & + & - & - & - & No & $6 / 9$ & $6 / 9$ & $6 / 9$ & $6 / 18$ & $6 / 12$ & $6 / 9$ & $14 / 1714 / 17$ & 6 & Green & D ref \\
\hline 10 & $66 \mathrm{M}$ & 44 & Yes & None & 180 & light-am & + & - & - & - & No & $6 / 6$ & $6 / 6$ & $6 / 5$ & $6 / 6$ & $6 / 9$ & $6 / 5$ & $14 / 1714 / 17$ & 7 & Brown & PVD \\
\hline 11 & $65 \mathrm{M}$ & 45 & Yes & Reactolit & 60 & pm-bright & + & + & - & - & Yes & $6 / 6$ & $6 / 5$ & $6 / 5$ & $6 / 9$ & $6 / 9$ & $6 / 5$ & $14 / 1714 / 17$ & 8 & Grey & PVD \\
\hline $12 \mathrm{G}$ & $45 \mathrm{M}$ & 28 & Yes & Dark & 30 & light-pm & + & - & - & - & No & $6 / 6$ & $6 / 6$ & $6 / 5$ & $6 / 6$ & $6 / 5$ & $6 / 5$ & $14 / 1714 / 17$ & 9 & Brown & Floater \\
\hline 13 & $28 \mathrm{~F}$ & 11 & Yes & Minus1.0 & 30 & pm-light & + & + & - & - & Some & $6 / 5$ & $6 / 5$ & $6 / 4$ & $6 / 5$ & $6 / 5$ & $6 / 4$ & $14 / 1714 / 17$ & 8 & Brown & Floater \\
\hline 14 & $67 \mathrm{M}$ & 40 & Yes & None & 30 & sunny & + & - & - & - & No & $6 / 6$ & $6 / 5$ & $6 / 4$ & $6 / 9$ & $6 / 5$ & $6 / 5$ & $14 / 1714 / 17$ & 8 & Blue & Floater \\
\hline 15 & $57 \mathrm{~F}$ & 24 & Yes & $\begin{array}{l}\text { p2.00ds } \\
\text { p1.50ad }\end{array}$ & 45 & pm-light & + & - & - & - & No & $6 / 12$ & $6 / 6$ & $6 / 6$ & $6 / 12$ & $6 / 6$ & $6 / 6$ & $15 / 1715 / 17$ & 8 & Blue & Naevus \\
\hline 16 & $40 \mathrm{M}$ & 17 & Yes & $\begin{array}{l}\text { p1.50ds } \\
\text { p1.50ad }\end{array}$ & 60 & am-light & - & - & - & - & No & $6 / 5$ & $6 / 5$ & $6 / 5$ & $6 / 6$ & $6 / 6$ & $6 / 6$ & $14 / 1714 / 17$ & 8 & Blue & PVD \\
\hline 17 & $61 \mathrm{~F}$ & 30 & Yes & $\begin{array}{l}\text { p.2.25ds } \\
\text { p2.5ad }\end{array}$ & 45 & pm-dusk & + & - & - & - & No & $6 / 9$ & $6 / 9$ & $6 / 9$ & $6 / 9$ & $6 / 9$ & $6 / 9$ & $14 / 1714 / 17$ & 8 & Hazel & PVD \\
\hline 18 & $33 \mathrm{M}$ & 14 & No & None & 45 & pm-light & + & + & + & + & No & $6 / 6$ & $6 / 9$ & $6 / 6$ & $6 / 5$ & $6 / 6$ & $6 / 5$ & 14/17 14/17 & 7 & Brown & PVD \\
\hline 19 & $73 \mathrm{M}$ & 55 & Yes & None & 6 & am-light & + & - & - & - & No & $6 / 9$ & $6 / 9$ & $6 / 9$ & $6 / 12$ & $6 / 12$ & $6 / 9$ & $14 / 1714 / 17$ & 7 & Grey & Floater \\
\hline 20 & $59 \mathrm{M}$ & 26 & Yes & None & 45 & am-light & - & - & - & - & No & $6 / 9$ & $6 / 5$ & $6 / 5$ & $6 / 9$ & $6 / 6$ & $6 / 6$ & $14 / 1714 / 17$ & 6 & Brown & Floater \\
\hline 21 & $70 \mathrm{M}$ & 46 & Yes & $\begin{array}{l}\text { p2.50ds } \\
\text { p2.00ad }\end{array}$ & 45 & am-light & - & - & - & - & No & $6 / 6$ & $6 / 6$ & $6 / 6$ & $6 / 6$ & $6 / 6$ & $6 / 6$ & $15 / 1715 / 17$ & 7 & Blue & Floater \\
\hline 22 & $40 \mathrm{~F}$ & 23 & Yes & None & 40 & dark & - & - & + & - & No & $6 / 9$ & $6 / 12$ & $6 / 6$ & $6 / 9$ & $6 / 9$ & $6 / 6$ & $15 / 1715 / 17$ & 7 & Brown & NAD \\
\hline 23 & $68 \mathrm{M}$ & 50 & Yes & None & 60 & pm-dark & - & - & - & - & No & $6 / 4$ & $6 / 6$ & $6 / 5$ & $6 / 6$ & $6 / 6$ & $6 / 5$ & $\mathrm{col} \mathrm{b} \mathrm{col} \mathrm{b}$ & 8 & Blue & PVD \\
\hline 24 & $57 \mathrm{M}$ & 34 & Yes & $\begin{array}{l}\text { p1.75ds } \\
\text { p1.25ad }\end{array}$ & 210 & pm-dark & + & - & - & - & No & $6 / 5$ & $6 / 6$ & $6 / 5$ & $6 / 6$ & $6 / 6$ & $6 / 5$ & $14 / 1414 / 14$ & 8 & Brown & PVD \\
\hline 25 & $62 \mathrm{~F}$ & 34 & No & $\begin{array}{l}\text { p2.75ds } \\
\text { p2.00ad }\end{array}$ & 120 & pm-dark & - & - & - & - & No & $6 / 9$ & $6 / 6$ & $6 / 6$ & $6 / 9$ & $6 / 9$ & $6 / 9$ & $14 / 1414 / 14$ & 7 & Blue & PVD \\
\hline 26 & $70 \mathrm{~F}$ & 43 & Yes & None & 45 & pm-light & - & - & - & - & No & $6 / 6$ & $6 / 6$ & $6 / 6$ & $6 / 5$ & $6 / 6$ & $6 / 6$ & - & 7 & Blue & PVD \\
\hline 27 & $20 \mathrm{~F}$ & 3 & Yes & None & 30 & am-light & + & - & - & - & No & $6 / 6$ & $6 / 5$ & $6 / 5$ & $6 / 9$ & $6 / 6$ & $6 / 6$ & $14 / 1414 / 14$ & 7 & Grey & Anisocoria \\
\hline 28 & $50 \mathrm{~F}$ & 22 & Yes & $\begin{array}{l}\text { p1.50ds } \\
\text { p1.25ad }\end{array}$ & 45 & pm-light & - & - & - & - & $\begin{array}{c}\text { Disoriented } \\
\text { a little }\end{array}$ & $6 / 6$ & $6 / 9$ & $6 / 5$ & $6 / 6$ & $6 / 12$ & $6 / 5$ & $13 / 1414 / 14$ & 5 & Blue-grey & $\begin{array}{c}\text { Peri-orbital } \\
\text { trauma }\end{array}$ \\
\hline 29 & $55 \mathrm{~F}$ & 35 & Yes & $\begin{array}{l}\text { p2.00ds } \\
\text { p1.50ad }\end{array}$ & 60 & pm-dark & - & - & - & - & No & $6 / 5$ & $6 / 5$ & $6 / 5$ & $6 / 9$ & $6 / 6$ & $6 / 6$ & $14 / 1414 / 14$ & 7 & Brown & Iritis \\
\hline 30 & $62 \mathrm{~F}$ & 30 & Yes & None & 60 & pm-dark & - & - & - & - & No & $6 / 6$ & $6 / 9$ & $6 / 6$ & $6 / 9$ & $6 / 9$ & $6 / 9$ & $14 / 1714 / 17$ & 7 & Brown & Floater \\
\hline
\end{tabular}

$\mathrm{PVD}$, posterior vitreous detachment; $\mathrm{D}$ ref, diabetic retinopathy screen; NAD, nil abnormal detected. 
will not suit all patients. Instead each patient must be assessed on an individual basis as to their ability to drive. The following guidelines may be helpful when advising a patient about driving with pharmacologically dilated pupils:

1. Test the patient's binocular visual acuity after the dilating drops have taken effect to make sure there is no drop in binocular visual acuity below 6/10.

2. Allow the patient to check in outdoor daylight conditions that he or she can read a registration plate $79.4 \mathrm{~mm}$ ( $3 \frac{1}{8}$ inches) high at $20.5 \mathrm{~m}$ and then drive if he or she feels comfortable doing so.

3. Advise the patient to drive only on familiar roads.
4. Advise the patient to allow time to adapt to the dilated pupil state (30-60 $\mathrm{min}$ ) before driving.

\section{References}

1. Munton G, Vision. In: Medical aspects of fitness to drive. London: The Medical Commission on Accident Prevention. 1995:118.

2. Drasdo M, Haggarty CM. A comparison of British number plates and Snellen vision tests for car driving. Research report RF 676. Crowthorne: Transport and Road Research Laboratory. 